\title{
A Performance Comparison of Robust Adaptive Controllers: Linear Systems
}

\author{
Ahmad Sanei* Mark French* \\ 31st March 2005 \\ Revised manuscript.
}

\begin{abstract}
We consider robust adaptive control designs for relative degree one, minimum phase linear systems of known high frequency gain. The designs are based on the dead-zone and projection modifications, and we compare their performance w.r.t. a worst case transient cost functional with a penalty on the $\mathcal{L}^{\infty}$ norm of the output, control and control derivative. We establish two qualitative results. If a bound on the $\mathcal{L}^{\infty}$ norm of the disturbance is known and the known a-priori bound on the uncertainty level is sufficiently conservative, then it is shown that a dead-zone controller outperforms a projection controller. The complementary result shows that the projection controller is superior to the dead-zone controller when the a-priori information on the disturbance level is sufficiently conservative.
\end{abstract}

Key words. Robust Adaptive Control, Non-singular Performance

\section{Introduction}

It is well known that adaptive controllers are suitable for systems whose nominal mathematical model contains an uncertain parameter $\theta$. A common feature of adaptive designs is the construction of a time varying parameter $\hat{\theta}(\cdot)$ whose value is controlled by an adaptive law. In contrast with most adaptive control mechanisms which would attempt to 'identify' or 'estimate' the uncertain parameter $\theta$ of the plant by a 'parameter estimator' $\hat{\theta}(\cdot)$, the objective of a 'non-identifier-based' adaptive controller is to use certain information about the plant to find suitable methods of system regulation. In other words, the adaptive law makes no attempt to identify or estimate the unknown plant parameter $\theta$, but merely attempts to seek out a stabilising value for the so-called 'tuning function' $\hat{\delta}(\cdot)$. See eg. [M, WB] and [I2] for an overview.

However, this method, like other adaptive controllers, is susceptible to phenomena such as parameter drift even when small disturbances are present. To overcome such problems, a number of standard techniques are widely utilised, such as dead-zones, $\sigma$ modification, projection modification, etc. [NA].

Each of these techniques has advantages and drawbacks. For example, dead-zone modifications typically require a-priori knowledge of the disturbance level, and only achieve convergence of the state/output/error to some pre-specified neighbourhood of the origin (whilst

${ }^{*}$ School of Electronics and Computer Science, University of Southampton, Southampton, SO17 1BJ, U.K. Telephone: +44(23) 8059 2688, Fax: +44(23) 8059 4498, Email: afs Imcf@ecs.soton.ac.uk 
keeping all signals bounded). In particular if the disturbance vanishes, then the dead-zone controller does not typically achieve convergence of the output to zero, the convergence remains to the pre-specified neighbourhood of the origin. On the other hand, projection modifications generally achieve boundedness of all signals, and furthermore have the desirable property that if no disturbances are present, then the state/output/error converges to zero, however, an arbitrarily small $\mathcal{L}^{\infty}$ disturbance can completely destroy any convergence to zero.

This illustrates that in the case of asymptotic performance, there are some known characterisations of 'good' and 'bad' behaviour. However, there are many situations in which we cannot definitively state whether a projection or dead-zone controller is superior. Although the asymptotic performance of such modifications has often been investigated, very little of this research is concerned with transient performance. Furthermore, the known results, see e.g. $[\mathrm{KKK}]$, as with most results in adaptive control, are confined to singular performance, i.e. without any consideration of the control signal. The primary results of this paper take control effort into account.

The present paper extends the line of our work on developing a comparison theory of robust adaptive controllers $[\mathrm{F}, \mathrm{SF}, \mathrm{XF}]$. We compare dead-zone and projection based adaptive controllers for finite dimensional, minimum phase, linear systems of relative degree one with positive high frequency gain. The comparison has been made with respect to a worst case nonsingular transient cost functional $\mathcal{P}$ penalising the state $(x)$, the input $(u)$ and the derivative of the input $(\dot{u})$ of the plant. We will identify circumstances in which the dead-zone controller is superior to the projection controller with respect to $\mathcal{P}$ and vice versa.

The paper is structured as follows. In Section 2 we introduce the system class and the basic adaptive controller. Section 3 defines two standard classes of robust modifications to the basic adaptive controller, namely the dead-zone modification and the projection modification, and states their main properties. The proofs of these results are deferred to Appendices A and B. Our main results are presented in Section 4, where situations are described in which the dead-zone controller outperforms the projection controller and vice-versa. Section 5 and 6 contain the proofs of the results of Section 4, and Section 7 contains a short discussion on alternative choices of dead-zones. Section 8 contains a brief summary and conclusions.

\section{System and Basic Control Design}

Suppose $\Sigma$ is a SISO linear time invariant plant described by

$$
y=\frac{b_{n-1} s^{n-1}+b_{n-2} s^{n-2}+\cdots+b_{0}}{s^{n}+a_{n-1} s^{n-1}+\cdots+a_{0}}(u+d)
$$

where $a_{i}, b_{i} \in \mathbb{R}, 0 \leq i \leq n-1$, are unknown constants and $d(\cdot)$ belongs to a class of bounded disturbances $\mathcal{D} \subset \mathcal{L}^{\infty}=\mathcal{L}^{\infty}\left[\mathbb{R}_{\geq 0}\right]=\mathcal{L}^{\infty}[0, \infty)$. Note that for notational convenience, we adopt the standard shorthand of letting $u, y, d$ denote both the time domain signal and corresponding element in frequency domain. We assume that only the output $y(\cdot)$ is available for measurement. Consider the following assumptions:

C1. The plant is minimum phase i.e. $b_{n-1} s^{n-1}+\cdots+b_{0}$ is Hurwitz.

C2. The plant order $n$ is known, and the high frequency gain is positive (i.e. $b_{n-1}>0$ ).

It is convenient for the analysis in this paper to consider two different state space represen- 
tation of (1). The observer canonical form is obtained as follows:

$$
\begin{aligned}
\Sigma\left(x_{0}, \theta, d(\cdot)\right): \quad \dot{x}(t) & =A x(t)+B(u(t)+d(t)) \quad x(0)=x_{0} \in \mathbb{R}^{n}, d \in \mathcal{D}, \\
y(t) & =C x(t),
\end{aligned}
$$

in which $x(t), B, C^{T} \in \mathbb{R}^{n}, A \in \mathbb{R}^{n \times n}$, and

$$
A=\left[\begin{array}{ccccc}
-a_{n-1} & 1 & 0 & \ldots & 0 \\
-a_{n-2} & 0 & 1 & 0 & 0 \\
\vdots & \vdots & \vdots & \ddots & \vdots \\
-a_{1} & 0 & 0 & \ldots & 1 \\
-a_{0} & 0 & 0 & \ldots & 0
\end{array}\right], B=\left[\begin{array}{c}
b_{n-1} \\
\vdots \\
b_{1} \\
b_{0}
\end{array}\right], C=\left[\begin{array}{llll}
1 & 0 & \cdots & 0
\end{array}\right]
$$

where

$$
\theta=\left(a_{0}, \ldots, a_{n-1}, b_{0}, \ldots, b_{n-1}\right) \in \mathcal{S}, \quad \mathcal{S}:=\left\{\theta \in \mathbb{R}^{2 n} \mid \Sigma(\cdot, \theta, \cdot) \text { satisfies } C 1, C 2\right\},
$$

and $\theta \in \mathcal{S}$ represents the uncertain system parameters. We emphasise that by non-identifierbased control, we will not be estimating unknown parameter $\theta$.

The second useful state space form is obtained by utilizing the coordinate transformation matrices:

$$
S:=\left[B(C B)^{-1}, T\right], \quad S^{-1}=\left[C^{T}, N^{T}\right]^{T},
$$

where

$$
T=\left[\begin{array}{c}
0 \ldots 0 \\
I_{n-1}
\end{array}\right] \in \mathbb{R}^{n \times(n-1)}, \quad N=\left[\begin{array}{cc}
-b_{n-2} / b_{n-1} & \\
\vdots & I_{n-2} \\
-b_{0} / b_{n-1} &
\end{array}\right] \in \mathbb{R}^{(n-1) \times n}
$$

Applying (5) to (2), we have

$$
\bar{x}(t):=\left(y(t), z(t)^{T}\right)^{T}=S^{-1} x(t), \quad S^{-1} B C S=\left[\begin{array}{cc}
b_{n-1} & 0 \\
0 & 0
\end{array}\right], \quad S^{-1} A S=\left[\begin{array}{cc}
\bar{a}_{1} & \bar{A}_{2} \\
\bar{A}_{3} & \bar{A}_{4}
\end{array}\right],
$$

where $\bar{a}_{1} \in \mathbb{R}, \bar{A}_{2}^{T}, \bar{A}_{3} \in \mathbb{R}^{n-1}$ and $\bar{A}_{4} \in \mathbb{R}^{(n-1) \times(n-1)}$. This gives

$$
\begin{array}{ll}
\dot{y}(t)=\bar{a}_{1} y(t)+\bar{A}_{2} z(t)+b_{n-1}(d(t)+u(t)), & y(0)=C x_{0}, \\
\dot{z}(t)=\bar{A}_{3} y(t)+\bar{A}_{4} z(t), & z(0)=N x_{0} .
\end{array}
$$

It has been shown that $\bar{A}_{4}$ is stable [I2], i.e. there exists a positive definite matrix $R=R^{T}>0$ satisfying the Lyapunov equation

$$
R \bar{A}_{4}+\bar{A}_{4}^{T} R=-I_{n-1} .
$$

Note that there exists $k_{\theta}^{*}>0$ such that for all $k \geq k_{\theta}^{*}$,

$$
\left(S^{-1}(A-k B C) S\right)^{T} P+P\left(S^{-1}(A-k B C) S\right) \leq-Q
$$

where the symmetric positive definite matrices $P, Q$ are defined as

$$
P=\left[\begin{array}{cc}
\frac{1}{2} & 0 \\
0 & R
\end{array}\right], \quad Q=\left[\begin{array}{cc}
1 & 0 \\
0 & \frac{1}{2} I_{n-1}
\end{array}\right]
$$


This can be shown by observing that for all $k>k_{\theta}^{*}$ and for all $\bar{x} \in \mathbb{R}^{n}$,

$$
\begin{aligned}
\bar{x}^{T}\left(\left(S^{-1}(A-k B C) S\right)^{T} P\right. & \left.+P\left(S^{-1}(A-k B C) S\right)\right) \bar{x} \\
& =-\left(b_{n-1} k-\bar{a}_{1}\right) y^{2}+y\left(\bar{A}_{2}+2 \bar{A}_{3}^{T} R\right) z-\|z\|^{2} \\
& \leq-\left(b_{n-1} k-M\right) y^{2}-\frac{1}{2}\|z\|^{2} \\
& \leq-\bar{x}^{T} Q \bar{x}
\end{aligned}
$$

where

$$
M:=\left|\bar{a}_{1}\right|+\left(\left\|\bar{A}_{2}\right\|+2\|R\|\left\|\bar{A}_{3}\right\|\right)^{2} / 2, \quad k_{\theta}^{*}:=(M+1 / 2) / b_{n-1} .
$$

Therefore, by considering the Lyapunov function $V(\bar{x})=\bar{x}^{T} P \bar{x}$, it follows that if $\theta \in \mathcal{S}$ then the system (2) is high-gain stabilizable, i.e. it is stabilized by the feedback $u=-k y$ for sufficient large $k$.

It was shown by Willems \& Byrnes [WB] that disturbance free $(\mathcal{D}=\{0\})$ systems of the form (2), i.e. $\Sigma\left(x_{0}, \theta, d(\cdot)\right)$ which satisfy $C 1, C 2$, are stabilised by the following simple adaptive high-gain controller:

$$
\begin{aligned}
\Xi: \quad u(t) & =-\hat{\delta}(t) y(t), \\
\dot{\hat{\delta}}(t) & =y(t)^{2} \quad \hat{\delta}(0)=0 .
\end{aligned}
$$

Special features of this strategy are its simplicity and the absence of any plant identification mechanism. This idea formed the foundation of the theory of 'universal adaptive control' which has continued to develop since 1985 in the areas of linear systems, nonlinear systems, and infinite dimensional systems, see for example [T, IT, R, LT] for some representative papers, and [I1] for an early survey. These controllers have also been considered for application studies [LK, GI].

\section{Robust Modifications to the Control Design}

For systems of the form (2), it is well known that even a small $\mathcal{L}^{\infty}$ disturbance can cause a drift of the tunable function $\hat{\delta}(\cdot)$, see e.g. [E, NA]. To overcome this problem, two distinct approaches have been proposed [NA]: $(i)$ using an appropriately rich reference input, and (ii) modifying the adaptation law. In this section we briefly explain two common methods in modifying the adaptive law, in particular dead-zone and parameter projection modifications (see e.g. [NA] for details).

Whilst a subset of results on asymptotic behaviour of dead-zone and projection modifications presented in this section is known, we remark that the boundedness properties of the closed loop signals (properties D2 and P2 of Theorems 3.1 and 3.2) do not appear in the literature, to the best knowledge of the authors. Moreover these properties play a key role in the main results.

\subsection{Dead-zone Modification}

Consider the unmodified adaptive law of the form $\dot{\hat{\delta}}(t)=y(t)^{2}, \hat{\delta}(0)=0$. The idea of the dead-zone is to modify the adaptive law so that the adaptive mechanism is 'switched off' when system output $y(\cdot)$ lies inside a region where the disturbance has a destabilising 
effect on the dynamics $[\mathrm{PN}]$. A-priori knowledge of the size of the disturbance is typically used to define the size of the dead-zone. Let $d_{\max }$ be the a-priori known upper bound of the disturbance level, i.e. $d_{\max } \geq\|d(\cdot)\|_{\mathcal{L}^{\infty}}$ for all $d(\cdot) \in \mathcal{D}$. For SISO output feedback systems (2), the standard definition of the modified adaptive law is

$$
\dot{\hat{\delta}}(t)=y(t)^{2} \tilde{D}_{\eta}(y(t)), \quad \hat{\delta}(0)=0, \quad \eta:=d_{\max }>0
$$

where $\tilde{D}_{\eta}(y):=0$ if $y \in[-\eta, \eta]$ and $\tilde{D}_{\eta}(y):=1$ if $y \notin[-\eta, \eta], \eta>0$. It should be observed that there are other valid choices for $\eta$, see Section 7 where this is discussed further.

Note that the discontinuous switching activity of the above definition introduces a r.h.s. discontinuity in the related differential equations resulting in the need for a more delicate analysis. In this paper, we take the advantages of so-called 'smooth dead-zone' for which, the local existence and uniqueness of the solution of the corresponding closed loop system follows directly from the classical theory of differential equations. However, modulo these technical issues, the authors expect that similar results to those in this paper can also be obtained for the 'standard dead-zone' (12). The smooth dead-zone is defined by

$$
D_{\eta}(y)= \begin{cases}0, & y \in[-\eta, \eta] \\ |y|-\eta, & y \notin[-\eta, \eta]\end{cases}
$$

where $\eta>0$ and leads to the modified adaptive law of the form,

$$
\begin{array}{ll}
\Xi_{D}\left(d_{\max }\right): & u(t)=-\hat{\delta}(t) y(t) \\
& \dot{\hat{\delta}}(t)=|y(t)| D_{\eta}(y(t)), \quad \hat{\delta}(0)=0, \quad \eta:=d_{\max }>0 .
\end{array}
$$

The following theorem establishes the properties of such controllers:

Theorem 3.1. Consider the closed loop system $\left(\Sigma\left(x_{0}, \theta, d(\cdot)\right), \Xi_{D}\left(d_{\max }\right)\right)$ defined by $(2)-(4)$ and (14), where $x_{0} \in \mathbb{R}^{n}, \theta \in \mathcal{S}, d(\cdot) \in \mathcal{L}^{\infty}$ and $d_{\max } \geq 0$. Then the following properties hold:

D1. There exists a unique solution $(x(\cdot), \hat{\delta}(\cdot)): \mathbb{R}_{\geq 0} \rightarrow \mathbb{R}^{(n+1)}$.

D2. There exists a continuous function $M: \mathbb{R}^{n} \times \mathcal{S} \times \mathbb{R}_{\geq 0} \times \mathbb{R}_{\geq 0} \rightarrow \mathbb{R}_{\geq 0}$ such that the closed loop signals $x(\cdot), \hat{\delta}(\cdot), u(\cdot)$ satisfy

$$
\|x(\cdot)\|_{\mathcal{L}^{\infty}}+\|\hat{\delta}(\cdot)\|_{\mathcal{L}^{\infty}}+\|u(\cdot)\|_{\mathcal{L}^{\infty}} \leq M\left(x_{0}, \theta,\|d(\cdot)\|_{\mathcal{L}^{\infty}, d_{\max }}\right)
$$

D3. $\lim _{t \rightarrow \infty} \inf _{x \in\left[-d_{\max }, d_{\max }\right]}|y(t)-x|=0$.

Proof. See Appendix A.

\subsection{Projection Modification}

The projection modification $[\mathrm{KN}]$ is an alternative method to eliminate parameter drift by keeping the adaptive parameter within some a-priori defined bounds. In 'identifier-based' adaptive control, this can be accomplished by projecting the parameter estimator into a given compact convex set containing the true parameter vector. The general definition for such method can be found in e.g. [KKK, page 511]. In our 'non-identifier-based' case, the 
definition is as follows. Let $\delta_{\max }>k_{\theta}^{*}$ where $k_{\theta}^{*}$ is defined by (10). The modified adaptive law is defined by

$$
\dot{\hat{\delta}}(t)=\left\{\begin{array}{cl}
y(t)^{2} & \hat{\delta}(t)<\delta_{\max } \\
0 & \hat{\delta}(t) \geq \delta_{\max }
\end{array}\right.
$$

Given an output $y:[0, \omega) \rightarrow \mathbb{R}$, where $\omega \in[0, \infty]=[0, \infty) \cup\{\infty\}$, we define the projection controller as follows:

$$
\begin{aligned}
\Xi_{P}\left(\delta_{\max }\right): & u(t)=-\hat{\delta}(t) y(t) & & \\
& \dot{\hat{\delta}}(t)=y(t)^{2}, \quad \hat{\delta}(0)=0, & & \forall t \in\left[0, T_{m}\right), \\
\dot{\hat{\delta}}(t) & =0, & & \forall t \in\left[T_{m}, \infty\right),
\end{aligned}
$$

where $T_{m}=\inf \left\{t \in[0, \infty] \mid \hat{\delta}(t)=\delta_{\max }\right\}$. Note that $T_{m}$ is the first time instance that $\hat{\delta}$ hits the boundary $\delta_{\max }$, and after this time $\hat{\delta}$ remains at constant level $\delta_{\max }$ and hence the adaptive law in (16) coincides with (15). We denote the respective closed loop system by $\left(\Sigma\left(x_{0}, \theta, d(\cdot)\right), \Xi_{P}\left(\delta_{\max }\right)\right)$. The stability of the closed loop is examined in the following theorem.

Theorem 3.2. Consider the closed loop system $\left(\Sigma\left(x_{0}, \theta, d(\cdot)\right), \Xi_{P}\left(\delta_{\max }\right)\right)$ defined by $(2)$, (16), where $x_{0} \in \mathbb{R}^{n}, \theta \in \mathcal{S}, d(\cdot) \in \mathcal{L}^{\infty}$ and let $\delta_{\max }>k_{\theta}^{*}$ where $k_{\theta}^{*} \geq 0$ is given by (10). Then the following properties hold:

P1. There exists a solution $(x(\cdot), \hat{\delta}(\cdot)): \mathbb{R}_{\geq 0} \rightarrow \mathbb{R}^{(n+1)}$.

P2. Let $\mathcal{V}=\left\{\left(\theta, \delta_{\max }\right) \in \mathcal{S} \times \mathbb{R}_{>0} \mid k_{\theta}^{*}<\delta_{\max }\right\}$. Then there exists a continuous function $M: \mathbb{R}^{n} \times \mathcal{V} \times \mathbb{R}_{\geq 0} \rightarrow \mathbb{R}_{\geq 0}$ such that the closed loop signals $x(\cdot), \hat{\delta}(\cdot), u(\cdot)$ satisfy

$$
\|x(\cdot)\|_{\mathcal{L}^{\infty}}+\|\hat{\delta}(\cdot)\|_{\mathcal{L}^{\infty}}+\|u(\cdot)\|_{\mathcal{L}^{\infty}} \leq M\left(x_{0}, \theta, \delta_{\max },\|d\|\right) .
$$

Proof. See Appendix B.

\section{Statement of the Main Results}

A goal in control theory is to design control laws which achieve good performance for any member of a specified class of systems. Consider a system $\Sigma$ which belongs to the set of all admissible systems $\mathcal{S}^{*}$. The performance of a controller $\Xi$ is measured by a cost functional $J$ dependent on some measurable signals (state/output/input). In this paper, we are interested in a worst case scenario, i.e. a performance $\mathcal{P}$ which is defined over the power set of $\mathcal{S}^{*}$ and is formulated as a supremum of all possible costs. Furthermore, the non-singular performance measure will penalise the state $(x)$ and the input and its derivative $(u, \dot{u})$ of the plant; specifically we will consider cost functionals of the form:

$$
\mathcal{P}\left(\Sigma\left(\mathcal{X}_{0}(\gamma), \Lambda, \mathcal{D}(\epsilon)\right), \Xi\right)=\sup _{x_{0} \in \mathcal{X}_{0}(\gamma)} \sup _{\theta \in \Lambda} \sup _{d \in \mathcal{D}(\epsilon)}\left(\|x(\cdot)\|_{\mathcal{L}^{\infty}}+\|u(\cdot)\|_{\mathcal{L}^{\infty}}+\|\dot{u}(\cdot)\|_{\mathcal{L}^{\infty}}\right)
$$

where

$$
\begin{array}{rlrl}
\mathcal{X}_{0}(\gamma) & :=\left\{x_{0} \in \mathbb{R}^{n} \mid\left\|x_{0}\right\| \leq \gamma\right\}, & \gamma>0, \\
\mathcal{D}(\epsilon):=\left\{d(\cdot) \in \mathcal{L}^{\infty} \mid\|d(\cdot)\|_{\mathcal{L}^{\infty}} \leq \epsilon\right\}, & \epsilon \geq 0,
\end{array}
$$

and $\Lambda$ is any compact subset of $\Delta(\delta)$, where

$$
\Delta(\delta):=\left\{\theta \in \mathcal{S} \mid k_{\theta}^{*} \leq \delta\right\}, \quad \delta \geq 0,
$$


where $A, B, C$ are defined in (3) and $\theta$ is given by (4). Note that $\Delta(\delta)$ is not bounded, and that there are elements on the boundary of $\Delta(\delta)$ which do not satisfy $C 1, C 2$ and for which the closed loop is not stable, hence generating an infinite cost. Therefore the second supremum cannot be taken over $\Delta(\delta)$. This is reflected in the bounds obtained in Theorems 3.1 and 3.2 (see relations A-11, A-18, and B-5).

\subsection{Main Results}

Both designs considered require particular types of a-priori information on the system or disturbance environment. The projection based design requires an explicit a-priori upper bound $\delta_{\max }$ on the stabilizing value of $\delta$, whereas the dead-zone design requires an explicit a-priori bound $d_{\max }$ on the magnitude $\epsilon$ of the disturbance signals $d \in \mathcal{D}(\epsilon)$. We therefore consider the situations where either $\delta_{\max }$ or $d_{\max }$ are large with respect to the 'true' values $\delta$ or $\epsilon$ respectively. This corresponds to the cases where the control designer has made conservative choices for the projection/dead-zone levels.

The following theorems are the main results of the paper:

Theorem I. Consider the system $\Sigma\left(x_{0}, \theta, d(\cdot)\right)$ and the controllers $\Xi_{D}(\cdot)$ and $\Xi_{P}(\cdot)$ defined by (2), (14) and (16) respectively, where $x_{0} \in \mathbb{R}^{n}, \theta \in \mathcal{S}, d \in \mathcal{L}^{\infty}$. Let $\delta, \epsilon>0$ and $\emptyset \neq \Lambda \subset \Delta(\delta)$ be compact. Consider the transient performance cost functional (17). Then for all $d_{\max } \geq \epsilon$, there exists $\delta_{\max }^{*} \geq \delta$ such that for all $\delta_{\max } \geq \delta_{\max }^{*}$,

$$
\mathcal{P}\left(\Sigma\left(\mathcal{X}_{0}(\gamma), \Lambda, \mathcal{D}(\epsilon)\right), \Xi_{P}\left(\delta_{\max }\right)\right)>\mathcal{P}\left(\Sigma\left(\mathcal{X}_{0}(\gamma), \Lambda, \mathcal{D}(\epsilon)\right), \Xi_{D}\left(d_{\max }\right)\right)
$$

This theorem can be interpreted as stating that if the a-priori knowledge of the parametric uncertainty level $\delta_{\max }$ is sufficiently conservative $\left(\delta_{\max } \geq \delta_{\max }^{*}\right)$, then the dead-zone based design will out-perform the projection based design, see figure 1(left).
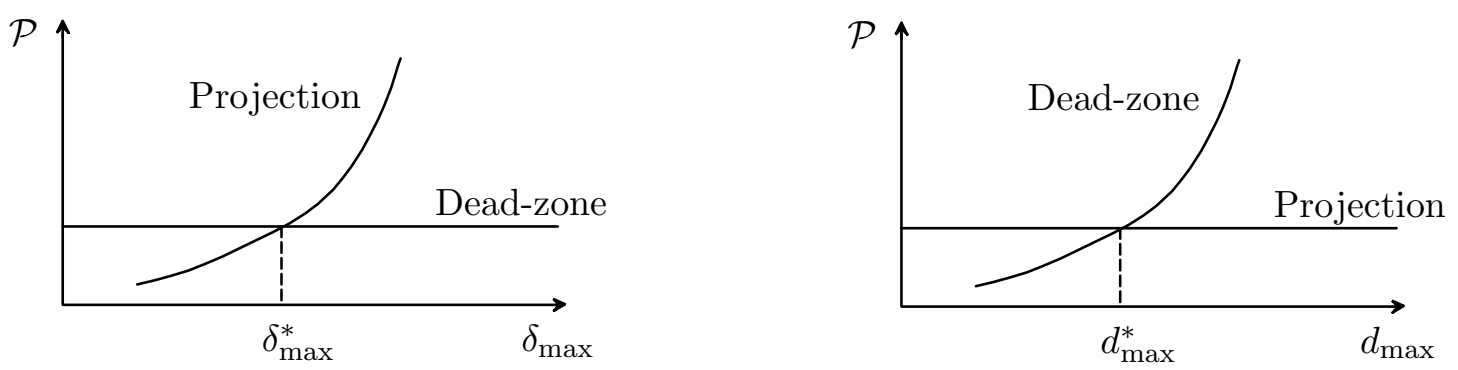

Figure 1: Statement of the main results: Theorem I (left), Theorem II (right)

Theorem II. Consider the system $\Sigma\left(x_{0}, \theta, d(\cdot)\right)$ and the controllers $\Xi_{D}(\cdot)$ and $\Xi_{P}(\cdot)$ defined by (2), (14) and (16) respectively, where $x_{0} \in \mathbb{R}^{n}, \theta \in \mathcal{S}, d \in \mathcal{L}^{\infty}$. Then for all $\epsilon>0$ 
there exists $\delta>0$ such that for all $\delta_{\max } \geq \delta$, there exists $d_{\max }^{*} \geq \epsilon>0$ such that for all $d_{\max } \geq d_{\max }^{*}$,

$$
\mathcal{P}\left(\Sigma\left(\mathcal{X}_{0}(\gamma), \Lambda, \mathcal{D}(\epsilon)\right), \Xi_{D}\left(d_{\max }\right)\right)>\mathcal{P}\left(\Sigma\left(\mathcal{X}_{0}(\gamma), \Lambda, \mathcal{D}(\epsilon)\right), \Xi_{P}\left(\delta_{\max }\right)\right),
$$

where $\mathcal{P}$ is the transient performance cost functional (17), $\Lambda \subset \Delta(\delta)$ is compact and $\Lambda \not \subset \Delta(0)$.

This theorem can be interpreted as stating that above a certain uncertainty level $\delta$, if the a-priori knowledge $d_{\max }$ of the disturbance level is sufficiently conservative $\left(d_{\max } \geq d_{\max }^{*}\right)$, then the projection design will out-perform the dead-zone design, see figure 1 (right).

As an aside, we observe that it is natural to ask whether there is any benefit of adaptive control when a bound $\delta_{\max }$ is known, as with this information, the natural static output feedback $u(t)=-\delta_{\max } y(t)$ achieves stabilization. One scenario in which adaptive strategies can be shown to outperform such memoryless feedbacks is when $\delta_{\max }$ is a conservative estimate for $\delta$, see, e.g. $[\mathrm{F}]$ for such an argument in a related context. In the context of this paper, similar arguments also show that one should utilize dead-zone adaptive controllers in preference to memoryless feedbacks in the scenario whereby $\delta_{\max }$ is a conservative estimate for $\delta$, and $d_{\max }$ is small. In the scenario where $d_{\max }$ is a conservative bound on $\epsilon$, the motivation for adaptation e.g. using the projection design over the memoryless design is less clear-cut. However, if both $\delta_{\max }$ and $d_{\max }$ are conservative estimates of $\delta$ and $\epsilon$ respectively, and classes of disturbances are considered in which adaptive law does not drift, (as for example in the disturbance free case), the projection design will in general not cause $\hat{\delta}$ to reach the projection value $\delta_{\max }$, and hence can be expected to generate a lower cost than the high gain memoryless design.

\section{Proof of Theorem I}

Firstly, we show that $\mathcal{P}=\infty$ for the unmodified design (11), (Proposition 5.3). From this we can show that the projection modification design, $\Xi_{P}\left(\delta_{\max }\right)$ has the property that $\mathcal{P} \rightarrow \infty$ as $\delta_{\max } \rightarrow \infty$ (Proposition 5.4). Finally we show that $\mathcal{P}<\infty$, and that the bound is independent of $\delta_{\max }$, for the dead-zone design, $\Xi_{D}\left(d_{\max }\right)$ (Proposition 5.5). This suffices to establish Theorem $\mathbf{I}$.

Proposition 5.1. Consider the closed loop system $\left(\Sigma\left(x_{0}, \theta, d(\cdot)\right), \Xi\right)$ defined by (2), (11), where $x_{0} \in \mathbb{R}^{n}, \theta \in \mathcal{S}$. Let $d(\cdot) \equiv \epsilon$ for some $\epsilon \neq 0$. Then

$$
\|x(t)\| \rightarrow 0 \text { as } t \rightarrow \infty \Longleftrightarrow \hat{\delta}(t) \rightarrow \infty \text { as } t \rightarrow \infty .
$$

Proof. Let $\left(x^{T}, \hat{\delta}\right)^{T}$ denote the unique global solution [WB] of the closed loop $\left(\Sigma\left(x_{0}, \theta, d(\cdot)\right), \Xi\right)$ given by equations (2), (11).

$\rightarrow$ ) Suppose for a contradiction that $\hat{\delta}(t) \nrightarrow \infty$. Then $\hat{\delta}(t) \rightarrow \hat{\delta}^{*}<\infty$, since $\hat{\delta}(\cdot)$ is monotonically increasing by (11). Therefore since by assumption $x \rightarrow 0$ and since the r.h.s. of $(2),(11)$ is continuous, it follows that $(x(\cdot), \hat{\delta}(\cdot)) \equiv\left(0, \hat{\delta}^{*}\right)$ is an equilibrium point of the closed loop $\left(\Sigma\left(x_{0}, \theta, d(\cdot)\right), \Xi\right)$. Hence $(x(\cdot), \hat{\delta}(\cdot)) \equiv\left(0, \hat{\delta}^{*}\right)$ must be a solution of the following equations:

$$
\begin{aligned}
x_{2}(t)-a_{n-1} x_{1}(t)+b_{n-1}\left(\epsilon-\hat{\delta}(t) x_{1}(t)\right) & =0, \\
\vdots & \\
-a_{0} x_{1}(t)+b_{0}\left(\epsilon-\hat{\delta}(t) x_{1}(t)\right) & =0 \\
x_{1}(t)^{2} & =0 .
\end{aligned}
$$


But $b_{0} \neq 0$ since the system is minimum phase. We also have $\epsilon \neq 0$. Therefore $(x(\cdot), \hat{\delta}(\cdot)) \equiv\left(0, \hat{\delta}^{*}\right)$ cannot be a solution of $(20)$, hence contradiction.

$\leftarrow) \quad$ Let $\zeta>0$ and recalling that $y=C x, z=N x$, define

$$
V(t)=\frac{1}{2} y(t)^{2}+z(t)^{T} R z(t), \quad t \geq 0,
$$

where $R$ is defined by equation 8 . The time derivative of $V$ along the solution of (7),(11) is

$$
\begin{aligned}
\dot{V}(t) & =y(t)\left(\left(\bar{a}_{1}-b_{n-1} \hat{\delta}(t)\right) y(t)+\bar{A}_{2} z(t)+b_{n-1} \epsilon\right)+2 y(t) \bar{A}_{3}^{T} R z(t)+z(t)^{T}\left(\bar{A}_{4}^{T} R+R \bar{A}_{4}\right) z(t) \\
& =-\left(b_{n-1} \hat{\delta}(t)-\bar{a}_{1}\right) y(t)^{2}+\frac{b_{n-1}}{\sqrt{\zeta}} y(t) \cdot \sqrt{\zeta} \epsilon+y(t)\left(\bar{A}_{2}+2 \bar{A}_{3}^{T} R\right) z(t)-\|z(t)\|^{2} \\
& \leq-\left(b_{n-1} \hat{\delta}(t)-M\right) y(t)^{2}-\frac{1}{2}\|z(t)\|^{2}+\frac{1}{2} \zeta \epsilon^{2},
\end{aligned}
$$

where $M:=\left|\bar{a}_{1}\right|+\frac{b_{n-1}^{2}}{2 \zeta}+\left(\left\|\bar{A}_{2}\right\|+2\|R\|\left\|\bar{A}_{3}\right\|\right)^{2} / 2$. We choose $t_{\zeta}>0$ such that $b_{n-1} \hat{\delta}(t)-$ $M>1 / 2$ for all $t \geq t_{\zeta}$. Therefore

$$
\dot{V}(t) \leq-\alpha V(t)+\frac{1}{2} \zeta \epsilon^{2} \quad \forall t \geq t_{\zeta}
$$

where $\alpha=\min \{1,1 / 2 \bar{\lambda}(R)\}$. It follows that

$$
\lim _{t \rightarrow \infty} \sup V(t) \leq \frac{\zeta \epsilon^{2}}{2 \alpha}
$$

Since $\zeta$ is arbitrary, this proves $\lim _{t \rightarrow \infty} V(t)=0$, hence $\lim _{t \rightarrow \infty}\left(y(t), z(t)^{T}\right)=0$. This completes the proof.

Proposition 5.2. Consider the closed loop system $\left(\Sigma\left(x_{0}, \theta, d(\cdot)\right), \Xi\right)$ defined by (2), (11), where $x_{0} \in \mathbb{R}^{n}, \theta \in \mathcal{S}$. Let $d(\cdot) \equiv \epsilon$ for some $\epsilon \neq 0$. If $x(\cdot)$ is uniformly continuous, then

$$
\|x(t)\| \rightarrow 0, \quad \hat{\delta}(t) \rightarrow \infty \quad \text { as } t \rightarrow \infty .
$$

Proof. Let $\left(x^{T}, \hat{\delta}\right)^{T}$ denote the unique global solution [WB] of the closed loop $\left(\Sigma\left(x_{0}, \theta, d(\cdot)\right), \Xi\right)$ given by equations (2), (11), and recall that $y=C x, z=N x$. Firstly we show that $y(t) \rightarrow 0$ as $t \rightarrow \infty$. From this we will prove that $\hat{\delta}(t) \rightarrow \infty$ and finally by Proposition 5.1, we conclude that $\|x(t)\| \rightarrow 0$ as $t \rightarrow \infty$.

Suppose for a contradiction that $y(t) \nrightarrow 0$ as $t \rightarrow \infty$. Then there exists $M>0$ and a positive sequence $\left\{t_{k}\right\}_{k \geq 1}, t_{k} \rightarrow \infty$ as $t \rightarrow \infty$ for which $y\left(t_{k}\right) \geq M$. Since, by assumption, $x(\cdot)$ is uniformly continuous, it follows that $y(\cdot)$ is uniformly continuous, and hence

$$
\exists \omega>0 \text { s.t. } \forall \tau \in[0, \omega], \text { and } \forall t>0, \quad|y(t)-y(t+\tau)|<\frac{M}{2} .
$$

Therefore $\left|y\left(t_{k}\right)-y\left(t_{k}+\tau\right)\right|<M / 2$ and since $y\left(t_{k}\right) \geq M$, we have that $y\left(t_{k}+\tau\right)>M / 2$ i.e. $y(t) \geq M / 2$ for all $t \in\left[t_{k}, t_{k}+\omega\right]$. Without loss of generality, we may assume $t_{k+1}-t_{k} \geq \omega$. It follows that

$$
\hat{\delta}\left(t_{k}+\omega\right)=\int_{0}^{t_{k}+\omega} \dot{\hat{\delta}}(\tau) d \tau=\int_{0}^{t_{k}+\omega} y^{2}(\tau) d \tau \geq \frac{M^{2}}{4} k \omega,
$$


so $\hat{\delta}\left(t_{k}+\omega\right) \rightarrow \infty$ as $k \rightarrow \infty$, hence $\hat{\delta}(t) \rightarrow \infty$ as $t \rightarrow \infty$. It follows by Proposition 5.1 that $\|x(t)\| \rightarrow 0$ as $t \rightarrow \infty$, therefore $y(t) \rightarrow 0$ as $t \rightarrow \infty$ by (2), hence contradiction.

Now we have $y(t)=x_{1}(t) \rightarrow 0$ as $t \rightarrow \infty$ and we claim $\hat{\delta}(t) \rightarrow \infty$ as $t \rightarrow \infty$. Suppose for contradiction $\hat{\delta}(t) \nrightarrow \infty$ as $t \rightarrow \infty$. Then $\hat{\delta}(t) \rightarrow \hat{\delta}^{*}<\infty$ as $t \rightarrow \infty$, since $\hat{\delta}(\cdot)$ is monotonic by (11). Substitute this into (2), we have

$$
\begin{aligned}
\dot{x}_{1}(t) & =x_{2}(t)-\left(a_{n-1}+\hat{\delta}^{*} b_{n-1}\right) x_{1}(t)+b_{n-1} \epsilon, \\
\vdots & \\
\dot{x}_{n}(t) & =-\left(a_{0}+\hat{\delta}^{*} b_{0}\right) x_{1}(t)+b_{0} \epsilon,
\end{aligned}
$$

where by minimum phase assumption, $b_{i} \epsilon \neq 0, i \in[0, n-1]$. Since $x_{1}(t) \rightarrow 0$ as $t \rightarrow \infty$, equation (24) implies that $x_{n}(t) \rightarrow \infty$ as $t \rightarrow \infty$, since $x(\cdot)$ is uniformly continuous. It follows that $x_{n-1}(t) \rightarrow \infty$ as $t \rightarrow \infty$, and cascading the argument shows to $x_{1}(t) \rightarrow \infty$ as $t \rightarrow \infty$, hence contradiction. Therefore $\hat{\delta}(t) \rightarrow \infty$ as $t \rightarrow \infty$. From this and Proposition 5.1, the claim of the Proposition follows.

Proposition 5.3. Consider the closed loop system $\left(\Sigma\left(x_{0}, \theta, d(\cdot)\right), \Xi\right)$ defined by (2), (11), where $x_{0} \in \mathbb{R}^{n}, \theta \in \mathcal{S}, d \in \mathcal{L}^{\infty}$. Let $\delta, \epsilon, \gamma>0$ and suppose $\emptyset \neq \Lambda \subset \Delta(\delta)$ is compact. Consider the transient performance cost functional (17). Then

$$
\mathcal{P}\left(\Sigma\left(\mathcal{X}_{0}(\gamma), \Lambda, \mathcal{D}(\epsilon)\right), \Xi\right)=\infty
$$

Proof. Let $\varlimsup$ denote $\limsup _{t \rightarrow \infty}$. Let $x_{0} \in \mathcal{X}_{0}(\gamma)$ and $\theta \in \Lambda$. Let $d_{1}(\cdot) \equiv \epsilon \neq 0$, and consider

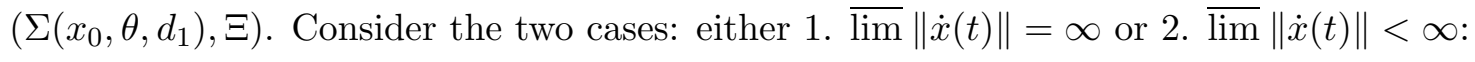

1. Suppose $\varlimsup\|\dot{\lim }\|(t) \|=\infty$, i.e. $\varlimsup \lim \|A x(t)+B u(t)+B \epsilon\|=\infty$. Therefore either

(a) $\varlimsup \lim \|x(t)\|=\infty$, which implies that $\|x(\cdot)\|_{\mathcal{L}^{\infty}}=\infty$, or

(b) $\varlimsup\|x\| x(t) \|<\infty$, therefore $\varlimsup u(t)=\infty$ i.e. $\|u(\cdot)\|_{\mathcal{L}^{\infty}}=\infty$.

2. Suppose $\varlimsup \lim \|\dot{x}(t)\|<\infty$ i.e. $x(\cdot)$ is uniformly continuous. Therefore by Proposition 5.2

$$
\forall \hat{\delta}^{*}>0 \quad \exists T>0 \text { s.t. } \forall t>T \quad \hat{\delta}(t)>\hat{\delta}^{*} .
$$

Now we choose $d_{2}(t):=\epsilon, \quad \forall t \leq T, d_{2}(t):=-\epsilon$, for all $t>T$. Note that $d_{2}(t)=d_{1}(t)$ for all $t \leq T$ and $d_{2} \in \mathcal{D}(\epsilon)$. Consider $\left(\Sigma\left(x_{0}, \theta, d_{2}\right), \Xi\right)$. With this choice, and by continuity and causality, we have that

$$
\lim _{t \rightarrow T^{+}} x(t)=x(T), \quad \lim _{t \rightarrow T^{+}} \hat{\delta}(t)=\hat{\delta}(T)
$$

where $\lim _{t \rightarrow T^{+}}$denote $\lim _{t \rightarrow T, t>T}$. It follows that

$$
\left(\lim _{t \rightarrow T^{+}} \dot{u}(t)\right)-\dot{u}(T)=2 \hat{\delta}(T) C B \epsilon \geq 2 \hat{\delta}^{*} b_{n-1} \epsilon .
$$

By choosing a suitable $\hat{\delta}^{*}$, it follows that $\hat{\delta}(T)$ can be made arbitrarily large and hence the difference (28) is arbitrarily large. Then either $\dot{u}(T)$ is large or $\lim _{t \rightarrow T^{+}} \dot{u}(t)$ is large, therefore $\|\dot{u}(\cdot)\|_{\mathcal{L}^{\infty}}$ can be made arbitrarily large.

Therefore at least one component of (17) is unbounded, hence

$$
\mathcal{P}\left(\Sigma\left(\mathcal{X}_{0}(\gamma), \Lambda, \mathcal{D}(\epsilon)\right), \Xi\right)=\infty
$$


Proposition 5.4. Consider the closed loop system $\left(\Sigma\left(x_{0}, \theta, d(\cdot)\right), \Xi_{P}\left(\delta_{\max }\right)\right)$ defined by $(2)$, (16), where $x_{0} \in \mathbb{R}^{n}, \theta \in \mathcal{S}, d \in \mathcal{L}^{\infty}$. Let $\delta, \epsilon, \gamma>0$ and suppose $\emptyset \neq \Lambda \subset \Delta(\delta)$ is compact. Consider the transient performance cost functional (17). Then

$$
\mathcal{P}\left(\Sigma\left(\mathcal{X}_{0}(\gamma), \Lambda, \mathcal{D}(\epsilon)\right), \Xi_{P}\left(\delta_{\max }\right)\right) \rightarrow \infty \quad \text { as } \quad \delta_{\max } \rightarrow \infty
$$

Proof. Let $M>0$. By Proposition 5.3 there exists $x_{0} \in \mathcal{X}_{0}(\gamma), d(\cdot) \in \mathcal{D}(\epsilon), \theta \in \Lambda$ so that the closed loop $\left(\Sigma\left(x_{0}, \theta, d(\cdot)\right), \Xi\right)$ satisfies

$$
\|x(\cdot)\|_{\mathcal{L}^{\infty}[0, \infty)}+\|u(\cdot)\|_{\mathcal{L}^{\infty}[0, \infty)}+\|\dot{u}(\cdot)\|_{\mathcal{L}^{\infty}[0, \infty)} \geq 2 M .
$$

It follows that $\exists T>0$ s.t. $\|x(\cdot)\|_{\mathcal{L}^{\infty}[0, T]}+\|u(\cdot)\|_{\mathcal{L}^{\infty}[0, T]}+\|\dot{u}(\cdot)\|_{\mathcal{L}^{\infty}[0, T]} \geq M$. By choosing $\delta_{\max }=2 \hat{\delta}(T)$, we have that $\delta_{\max }>\hat{\delta}(T)$, hence the solutions $\left(\Sigma\left(x_{0}, \theta, d(\cdot)\right), \Xi\right)$ and $\left(\Sigma\left(x_{0}, \theta, d(\cdot)\right), \Xi_{P}\left(\theta_{\max }\right)\right)$ are identical on $[0, T]$, and therefore

$$
\mathcal{P}\left(\Sigma\left(\mathcal{X}_{0}(\gamma), \Lambda, \mathcal{D}(\epsilon)\right), \Xi_{P}\left(\delta_{\max }\right)\right) \geq\|x(\cdot)\|_{\mathcal{L}^{\infty}[0, T]}+\|u(\cdot)\|_{\mathcal{L}^{\infty}[0, T]}+\|\dot{u}(\cdot)\|_{\mathcal{L}^{\infty}[0, T]} \geq M
$$

Since this holds for all $M>0$, this completes the proof.

Proposition 5.5. Consider the closed loop $\left(\Sigma\left(x_{0}, \theta, d(\cdot)\right), \Xi_{D}\left(d_{\max }\right)\right)$ defined by (2), (14), where $x_{0} \in \mathbb{R}^{n}, \theta \in \mathcal{S}, d \in \mathcal{L}^{\infty}$. Let $\delta, \epsilon, \gamma>0$ and suppose $\emptyset \neq \Lambda \subset \Delta(\delta)$ is compact. Consider the transient performance cost functional (17). Then

$$
\mathcal{P}\left(\Sigma\left(\mathcal{X}_{0}(\gamma), \Lambda, \mathcal{D}(\epsilon)\right), \Xi_{D}\left(d_{\max }\right)\right)<\infty, \quad \forall d_{\max }>\epsilon \geq 0
$$

Proof. Let $x_{0} \in \mathcal{X}_{0}(\gamma), \theta \in \Lambda$ and $d \in \mathcal{D}(\epsilon)$. A direct application of Property D2 of Theorem 3.1 guarantees the boundedness of $x(\cdot), \hat{\delta}(\cdot), u(\cdot)$ as a continuous function of $x_{0}, \theta, d_{\max }$. Since

$$
\dot{u}(t)=-y(t)^{2} D_{d_{\max }}(y(t))-\hat{\delta}(t) C((A-\hat{\delta}(t) B C) x(t)+B d(t)), \quad \forall t \geq 0
$$

it follows that $\|u(\cdot)\|_{\mathcal{L}^{\infty}}$ is bounded in terms of a continuous function of $x_{0}, \theta, d_{\text {max }}$. Therefore

$$
\mathcal{P}\left(\Sigma\left(x_{0}, \theta, d(\cdot)\right), \Xi_{D}\left(d_{\max }\right)\right) \leq M\left(x_{0}, \theta, d_{\max }\right),
$$

for some continuous function $M: \mathbb{R}^{n} \times \mathcal{S} \times \mathbb{R}_{\geq 0} \rightarrow \mathbb{R}_{\geq 0}$. Taking the supremum over system parameters $x_{0}, \theta, d$ implies that for all $d_{\max } \geq \epsilon$,

$$
\mathcal{P}\left(\Sigma\left(\mathcal{X}_{0}(\gamma), \Lambda, \mathcal{D}(\epsilon)\right), \Xi_{D}\left(d_{\max }\right)\right) \leq \sup _{x_{0} \in \mathcal{X}_{0}(\gamma)} \sup _{\theta \in \Lambda} \sup _{d \in \mathcal{D}(\epsilon)} M\left(x_{0}, \theta, d_{\max }\right)<\infty .
$$

Proof of Theorem $\boldsymbol{I}$.

This is a simple consequence of Proposition 5.4 and Proposition 5.5.

\section{Proof of Theorem II}

In order to prove Theorem II, we first give the following Propositions:

Proposition 6.1. Consider the closed loop system $\left(\Sigma\left(x_{0}, \theta, d(\cdot)\right), \Xi_{D}\left(d_{\max }\right)\right)$ defined by $(2)$, (14), where $x_{0} \in \mathbb{R}^{n}, \theta \in \mathcal{S}, d \in \mathcal{L}^{\infty}$. Let $\delta, \epsilon, \gamma>0$ and suppose $\emptyset \neq \Lambda \subset \Delta(\delta)$ is compact, and $\Lambda \not \subset \Delta(0)$. Consider the transient performance cost functional (17). Then $\exists \delta>0$ such that

$$
\mathcal{P}\left(\Sigma\left(\mathcal{X}_{0}(\gamma), \Lambda, \mathcal{D}(\epsilon)\right), \Xi_{D}\left(d_{\max }\right)\right) \rightarrow \infty, \quad \text { as } \quad d_{\max } \rightarrow \infty
$$


Proof. Since $\Lambda \not \subset \Delta(0)$, there exists $\theta \in \Lambda$ such that $A$ has an eigenvalue with strictly positive real part or a purely imaginary eigenvalue. In either case it follows that there exists $x_{0} \in \mathcal{X}_{0}(\gamma) \cap[-\eta, \eta]$ and $d \in \mathcal{D}(\epsilon)$ such that limsup $|\tilde{x}(t)|=\infty$ where $\tilde{x}(\cdot)$ is the solution to:

$$
\dot{\tilde{x}}(t)=A \tilde{x}(t)+B d(t) \quad \tilde{x}(0)=x_{0} \quad t \in[0, \infty) .
$$

Consider the closed loop system $\left(\Sigma\left(x_{0}, \theta, d(\cdot)\right), \Xi_{D}\left(d_{\max }\right)\right)$ and define $\tau$ as follows:

$$
\tau=\left\{\begin{array}{cl}
\infty & \text { if } y(t) \in[-\eta, \eta] \forall t \geq 0 \\
\inf \{t \geq 0 \mid & \text { otherwise. }
\end{array}\right.
$$

Note that by dead-zone definition $(14), \dot{\hat{\delta}}(t)=0$ for all $t \in[0, \tau)$, hence $\hat{\delta}(t)=0$ for all $t \in[0, \tau)$ since $\hat{\delta}(0)=0$. Therefore

$$
\dot{x}(t)=A x(t)+B d(t) \quad x(0)=x_{0} \quad \forall t \in[0, \tau) .
$$

Since $x(t)=\tilde{x}(t)$ for $t \in[0, \tau)$ it follows by the observability of $(A, C)$ that $y(t)=C x(t)$ hits the boundary of $[-\eta, \eta]$ in finite time, i.e. $\tau<\infty$. It follows that

$$
\|x(\cdot)\|_{\mathcal{L}^{\infty}} \geq|y(\tau)|=\eta=d_{\max } .
$$

Hence $\mathcal{P}\left(\Sigma\left(x_{0}, \theta, d(\cdot)\right), \Xi_{D}\left(d_{\max }\right)\right) \rightarrow \infty$ as $d_{\max } \rightarrow \infty$, and therefore

$$
\mathcal{P}\left(\Sigma\left(\mathcal{X}_{0}(\gamma), \Lambda, \mathcal{D}(\epsilon)\right), \Xi_{D}\left(d_{\max }\right)\right) \rightarrow \infty \quad \text { as } d_{\max } \rightarrow \infty
$$

Proposition 6.2. Consider the closed loop $\left(\Sigma\left(x_{0}, \theta, d(\cdot)\right), \Xi_{P}\left(\delta_{\max }\right)\right)$ defined by $(2),(16)$, where $x_{0} \in \mathbb{R}^{n}, \theta \in \mathcal{S}, d \in \mathcal{L}^{\infty}$. Let $\delta, \epsilon, \gamma>0$ and suppose $\emptyset \neq \Lambda \subset \Delta(\delta)$ is compact. Then for all $\delta_{\max } \geq \delta$,

$$
\mathcal{P}\left(\Sigma\left(\mathcal{X}_{0}(\gamma), \Lambda, \mathcal{D}(\epsilon)\right), \Xi_{P}\left(\delta_{\max }\right)\right)<\infty
$$

Proof. Let $x_{0} \in \mathcal{X}_{0}(\gamma), \theta \in \Lambda, d \in \mathcal{D}(\epsilon)$. A direct application P2 of Theorem 3.2 guarantees the boundedness of signals $x(\cdot), \hat{\delta}(\cdot), u(\cdot)$ of the closed loop $\left(\Sigma\left(x_{0}, \theta, d(\cdot)\right), \Xi_{P}\left(\delta_{\max }\right)\right)$ as a continuous function of $x_{0}, \theta,\|d\|, \delta_{\max }$. It follows that

$$
\dot{u}(t)=-y(t)^{3}-\hat{\delta}(t) C(A x(t)+B(u(t)+d(t)))
$$

is bounded in terms of a continuous function of $x_{0}, \theta,\|d\|, \delta_{\max }$, hence

$$
\mathcal{P}\left(\Sigma\left(x_{0}, \theta, d(\cdot)\right), \Xi_{P}\left(\delta_{\max }\right)\right) \leq M\left(x_{0}, \theta, \delta_{\max },\|d\|\right)<\infty,
$$

where $M: \mathbb{R}^{n} \times \mathcal{V} \times \mathbb{R}_{\geq 0} \rightarrow \mathbb{R}_{\geq 0}$ is continuous. Taking the supremum over system parameters $x_{0}, \theta, d$ implies that for all $\delta_{\max } \geq \delta$

$$
\mathcal{P}\left(\Sigma\left(\mathcal{X}_{0}(\gamma), \Lambda, \mathcal{D}(\epsilon)\right), \Xi_{P}\left(\delta_{\max }\right)\right) \leq \sup _{x_{0} \in \mathcal{X}_{0}(\gamma)} \sup _{\theta \in \Lambda} \sup _{d \in \mathcal{D}(\epsilon)} M\left(x_{0}, \theta, \delta_{\max },\|d\|\right)<\infty .
$$

\section{Proof of Theorem II.}

This is a simple consequence of Proposition 6.2 and Proposition 6.1.

The proof of Theorem II is heavily based on the natural assumption that the size of dead-zone $\eta$ is chosen to be equal to the a-priori bound on the disturbance level $d_{\max }$. In particular, $\eta:=d_{\max }$ implies that $\mathcal{P}\left(\Sigma\left(\mathcal{X}_{0}(\gamma), \Delta(\delta), \mathcal{D}(\epsilon)\right), \Xi_{D}\left(d_{\max }\right)\right) \rightarrow \infty$ as $d_{\max } \rightarrow \infty$. In the following section we show that the other choices of $\eta$ also yield the similar results. 


\section{Alternative Choices of Dead-zones}

In this section we consider alternative choices for the dead-zone, and variations on the definition of the controller. We consider the following smooth dead-zone controller (compare to equation (14)):

$$
\begin{aligned}
& \Xi_{D, \varrho}\left(d_{\max }\right):u(t)=-\hat{\delta}(t) y(t)) \\
& \dot{\hat{\delta}}(t)=|y(t)| D_{\eta}(y(t)), \quad \hat{\delta}(0)=0, \quad \eta:=\varrho\left(d_{\max }\right)
\end{aligned}
$$

where $\varrho:[0, \infty) \rightarrow[0, \infty)$, noting that we have previously considered the case where $\varrho(r)=r$. We consider two possibilities for the growth of the function $\varrho:{ }^{1}$

(i) $\lim _{r \rightarrow \infty} \varrho(r)=\infty$,

(ii) $\lim _{r \rightarrow \infty} \varrho(r) \leq c$ for some constant $c \geq 0$.

In case (i) it is straightforward to observe that

$$
\mathcal{P}:=\mathcal{P}\left(\Sigma\left(\mathcal{X}_{0}(\gamma), \Lambda, \mathcal{D}(\epsilon)\right), \Xi_{D, \varrho}\left(d_{\max }\right)\right) \rightarrow \infty \quad \text { as } d_{\max } \rightarrow \infty,
$$

by the same argument as in Proposition 6.1.

Choice (ii) is also a viable option, since $\Xi_{D, \varrho}\left(d_{\max }\right)=\Xi_{D}\left(\varrho\left(d_{\max }\right)\right)$, and by Theorem 3.1 , $\mathcal{P}\left(\Sigma\left(\mathcal{X}_{0}(\gamma), \Lambda, \mathcal{D}(\epsilon)\right), \Xi_{D, \varrho}\left(d_{\max }\right)\right)<\infty$ and $\lim _{t \rightarrow \infty} \inf _{x \in\left[-\varrho\left(d_{\max }\right), \varrho\left(d_{\max }\right)\right]}|y(t)-x|=0$. This choice has been extensively studied in the context of tracking, where the case $\varrho(r)=\lambda$ is known as $\lambda$-tracking [IR]. The next result shows that if $c$ is too small, then the projection controller out-performs the dead-zone design. For simplicity we consider a scalar system:

Proposition 7.1. Consider the system $\Sigma\left(x_{0},(a, 1), d(\cdot)\right)$ and the controllers $\Xi_{D, \varrho}(\cdot)$ and $\Xi_{P}(\cdot)$ defined by $(2)$, (32) and (16) respectively, where $x_{0} \in \mathbb{R}, a \in \mathbb{R}, d \in \mathcal{L}^{\infty}$ and where $\lim _{r \rightarrow \infty} \varrho(r) \leq c$ for $c>0$. Let $\delta, \epsilon>0$ and let $\Lambda=\left\{(a, 1) \in \mathbb{R}^{2}:|a| \leq \delta\right\}$. Consider the transient performance cost functional (17). Then for all $d_{\max } \geq \epsilon$, and for all $\delta_{\max } \geq \delta$, there exists $c^{*}>0$ such that for all $0<c<c^{*}$,

$$
\mathcal{P}\left(\Sigma\left(\mathcal{X}_{0}(\gamma), \Lambda, \mathcal{D}(\epsilon)\right), \Xi_{D, \varrho}\left(d_{\max }\right)\right)>\mathcal{P}\left(\Sigma\left(\mathcal{X}_{0}(\gamma), \Lambda, \mathcal{D}(\epsilon)\right), \Xi_{P}\left(\theta_{\max }\right)\right) .
$$

Proof. Let $x_{0} \in \mathcal{X}_{0}(\gamma)$ and suppose $(a, 1) \in \Lambda$ is such that $a>0$. Let $d(\cdot)=\epsilon>0$ be constant. Consider $\left(\Sigma\left(x_{0},(a, 1), d\right), \Xi_{D, \varrho}\left(d_{\max }\right)\right)$. Since $y(t) \rightarrow\left[-\varrho\left(d_{\max }\right), \varrho\left(d_{\max }\right)\right]$ as $t \rightarrow \infty$, there exists $T>0$ such that $y(T) \leq 2 \varrho\left(d_{\max }\right)$ and $\frac{1}{2} \frac{d}{d t} y(T)^{2}=\dot{y}(T) y(T) \leq 0$. It follows that,

$$
(\hat{\delta}(T)-a) \geq \frac{\epsilon}{2 \varrho\left(d_{\max }\right)} .
$$

Define $d_{2}(\cdot)$ as in Proposition 5.3 and consider $\left(\Sigma\left(x_{0}, \theta, d_{2}\right), \Xi_{D, \varrho}\left(d_{\max }\right)\right)$. It follows that

$$
\left(\lim _{t \rightarrow T^{+}} \dot{u}(t)\right)-\dot{u}(T)=2 \hat{\delta}(T) \epsilon=\frac{\epsilon^{2}}{\varrho\left(d_{\max }\right)} \geq \frac{\epsilon^{2}}{c} .
$$

Hence for all $\mathcal{P}^{*}>0$, there exists $c^{*}>0$ such that for all $0<c<c^{*}$,

$$
\begin{aligned}
\mathcal{P}\left(\Sigma\left(\mathcal{X}_{0}(\gamma), \Lambda, \mathcal{D}(\epsilon)\right), \Xi_{D, \varrho}\left(d_{\max }\right)\right) & =\sup _{x_{0} \in \mathcal{X}_{0}(\gamma)} \sup _{\theta \in \Lambda} \sup _{d \in \mathcal{D}(\epsilon)}\|\dot{u}(\cdot)\|_{\mathcal{L}^{\infty}} \\
& >\mathcal{P}^{*} .
\end{aligned}
$$

The proof is completed by choosing $\mathcal{P}^{*}=\mathcal{P}\left(\Sigma\left(\mathcal{X}_{0}(\gamma), \Lambda, \mathcal{D}(\epsilon)\right), \Xi_{P}\left(\theta_{\max }\right)\right)<\infty$.

\footnotetext{
${ }^{1}$ Other cases such as oscillatory but unbounded $\varrho(\cdot)$ can be handled suitably by considering monotonic subsequences.
} 
Similar results can be obtained in the tracking setting, where the error signal $e(\cdot)=$ $y(\cdot)-y_{\text {ref }}(\cdot)$ replaces the state $x(\cdot)=y(\cdot)$ and $\dot{y}_{\text {ref }}(\cdot)-\theta y_{\text {ref }}(\cdot)$ plays an analogous role to the disturbance $d(\cdot)$. See $[\mathrm{S}],[\mathrm{SF}]$ for further discussions.

\section{Conclusion}

In this paper we have established two rigorous results to demonstrate situations in which we can compare the transient performance of projection and dead-zone based controllers of non identifier based adaptive designs. We have shown that

- The dead-zone based controller outperforms the projection based controller when the a-priori information on the uncertainty level is sufficiently conservative.

- The projection based controller outperforms the dead-zone based controller when the a-priori information on the disturbance level is sufficiently conservative.

Our results are based on the a-priori information $d_{\max }$ and $\theta_{\max }$. We have also shown that other choices of controllers with differing dependence on a-priori information such as $\lambda$-tracking controllers can be driven to similar conclusions.

There are a number of directions in which the result can be generalised, for example: ( $i$ ) generalisation of the result for adaptive controllers for higher relative degree plants, (ii) generalisation of the results for nonlinear systems in the form of integrator chain $[S]$, or in the strict feedback form, and (iii) establishing whether the same results can be given for the alternative costs, for example, $\mathcal{P}=\|x(\cdot)\|_{\mathcal{L}^{\infty}}+\|u(\cdot)\|_{\mathcal{L}^{\infty}}$.

It would also be of interest to determine explicit tight bounds on $d_{\max }^{*}, \delta_{\max }^{*}$, note however that this is challenging as it would require tight upper and lower bounds on performance of both controllers.

Acknowledgements. We would like to express our gratitude to the reviewers for their perceptive comments and detailed reading of the draft manuscript.

\section{Appendices}

The proofs of Theorems 3.1 and 3.2 are given in this part.

\section{Appendix A. Proof of Theorem 3.1}

Before we give the proof, we first present a preliminary Lemma:

Lemma A-1. Suppose $M \in \mathbb{R}^{n \times n}, n \geq 1$ is an stable matrix. Let the positive definite matrix $G \in \mathbb{R}^{n \times n}$ be the solution of the Lyapunov equation

$$
G M+M^{T} G=-I .
$$

Then for all $t \geq 0$,

$$
\left\|e^{M t}\right\| \leq k_{1} e^{-k t}, \quad k:=1 / \bar{\lambda}(G), \quad k_{1}:=\sqrt{\bar{\lambda}(G) / \underline{\lambda}(G)},
$$

where $\underline{\lambda}(G), \bar{\lambda}(G)$ denote the minimum and maximum eigenvalue of $G$ respectively. 
Proof. The proof is elementary and omitted for brevity.

The proof of Theorem 3.1 is derived from the technique used in the proof of ' $\lambda$-tracking' based on [I2](pages 112-117) but with a significant extension to obtain property D2.

Proof of Theorem 3.1. The continuity of $D_{\eta}$ implies by classical result of differential equations that there exists a unique solution $(x(\cdot), \hat{\delta}(\cdot))$ over a maximal interval of existence $[0, \omega)$ for some $\omega \in(0, \infty]$. Using the transformation defined by $(5)$, we obtain

$$
\begin{array}{lrl}
\dot{y}(t)=\left(\bar{a}_{1}-b_{n-1} \hat{\delta}(t)\right) y(t)+\bar{A}_{2} z(t)+b_{n-1} d(t), & y(0)=C x_{0}, \\
\dot{z}(t)=\bar{A}_{3} y(t)+\bar{A}_{4} z(t), & z(0)=N x_{0}, \\
\dot{\hat{\delta}}(t)=|y(t)| D_{d_{\max }}(y(t)), & \hat{\delta}(0)=0
\end{array}
$$

Define the $\mathcal{C}^{1}$ function

$$
V(y):= \begin{cases}0, & y \in[-\eta, \eta], \\ \frac{1}{2}(|y|-\eta)^{2}, & y \notin[-\eta, \eta],\end{cases}
$$

where $\eta=d_{\max } \geq 0$. Observe that

$$
\dot{V}(y(t))=\zeta(t) \dot{y}(t) \quad \forall t \in[0, \omega)
$$

where the continuous function $\zeta:[0, \omega) \rightarrow \mathbb{R}$ is

$$
\zeta(t):=\left\{\begin{array}{cl}
0, & y(t) \in[-\eta, \eta] . \\
(|y(t)|-\eta) \frac{y(t)}{|y(t)|}, & y(t) \notin[-\eta, \eta] .
\end{array}\right.
$$

Note that by (14)

$$
\dot{\hat{\delta}}(t)=\zeta(t) y(t)=|\zeta(t)||y(t)|, \quad \forall t \in[0, \omega)
$$

and by the continuity of (A-6), $|y(t)||\zeta(t)| \geq \eta|\zeta(t)|$, or

$$
|\zeta(t)| \leq \eta^{-1}|\zeta(t)||y(t)|=\eta^{-1} \dot{\hat{\delta}}(t), \quad \forall t \in[0, \omega)
$$

Substituting (A-1) in (A-5), we have for all $t \in[0, \omega)$,

$$
\begin{aligned}
\dot{V}(y(t)) & =\left(\bar{a}_{1}-b_{n-1} \hat{\delta}(t)\right) y(t) \zeta(t)+\bar{A}_{2} z(t) \zeta(t)+b_{n-1} d(t) \zeta(t) \\
& \leq\left(\bar{a}_{1}-b_{n-1} \hat{\delta}(t)\right) \dot{\hat{\delta}}(t)+b_{n-1}\|d(\cdot)\|_{\mathcal{L}^{\infty}}|\zeta(t)|+\left\|\bar{A}_{2}\right\|\|z(t)\||\zeta(t)| \\
& \leq\left(M_{1}-b_{n-1} \hat{\delta}(t)\right) \dot{\hat{\delta}}(t)+\left\|\bar{A}_{2}\right\|\|z(t)\||\zeta(t)|,
\end{aligned}
$$

where $M_{1}:=M_{1}\left(\theta, d_{\max },\|d(\cdot)\|_{\mathcal{L}^{\infty}}\right):=\left|\bar{a}_{1}\right|+\eta^{-1} b_{n-1}\|d(\cdot)\|_{\mathcal{L}^{\infty}}$. Note that the continuous dependency of $M_{1}$ on $\theta \in \mathcal{S}$ follows from definition (4) and transformation (6) which also depends continuously on $\theta \in \mathcal{S}$. $M_{1}$ also depends continuously on $d_{\max }$ by definition (14).

Now we derive a relation between the second part of (A-9) and $\hat{\delta}(\cdot)$. Rewrite equation $(\mathrm{A}-2)$ as

$$
\dot{z}(t)=\bar{A}_{4} z(t)+\bar{A}_{3} \zeta(t)+h(t),
$$

where

$$
h(t):=\bar{A}_{3}(y(t)-\zeta(t))
$$


Note that since

$$
y-\zeta= \begin{cases}y, & |y|<\eta \\ \eta \frac{y}{|y|}, & |y| \geq \eta\end{cases}
$$

we have that $|h(t)| \leq \eta\left\|\bar{A}_{3}\right\|$. Since $\bar{A}_{4}$ is exponentially stable, by Lemma A-1, for all $t \in[0, \omega)$

$$
M_{2} e^{-\mu t} \geq\left\|e^{\bar{A}_{4} t}\right\|, \quad M_{2}:=M_{2}(\theta)=\sqrt{\frac{\overline{\bar{\lambda}}(R)}{\underline{\lambda}(R)}}, \quad \mu:=\mu(\theta)=1 / \bar{\lambda}(R),
$$

where $R$ is defined in (8). $M_{2}$ and $\mu$ depend continuously on $\theta \in \mathcal{S}$ since $\bar{A}_{4}$ depends continuously on $\theta \in \mathcal{S}$, hence $R$ and its eigenvalues are continuously dependent on $\theta \in \mathcal{S}$. Therefore by equation (A-10), we have for all $t \in[0, \omega)$

$$
\begin{aligned}
\|z(t)\| & \leq M_{2} e^{-\mu t}\left\|z_{0}\right\|+M_{2} \int_{0}^{t} e^{-\mu(t-s)}\left(\left\|\bar{A}_{3}\right\||\zeta(s)|+|h(s)|\right) d s \\
& \leq M_{2} e^{-\mu t}\left\|z_{0}\right\|+M_{2}\left\|\bar{A}_{3}\right\| \int_{0}^{t} e^{-\mu(t-s)}|\zeta(s)| d s+M_{2}\left\|\bar{A}_{3}\right\| \eta \mu^{-1}\left(1-e^{-\mu t}\right) \\
& \leq M_{3}\left(1+\int_{0}^{t} e^{-\mu(t-s)}|\zeta(s)| d s\right)
\end{aligned}
$$

where $M_{3}:=M_{3}\left(x_{0}, \theta\right):=M_{2}\left[\left\|z_{0}\right\|+\left\|\bar{A}_{3}\right\|\left(1+\eta \mu^{-1}\right)\right]$. The continuous dependency of $M_{3}$ on $x_{0}, \theta \in \mathcal{S}, d_{\max }$ follows from the definition of $\eta$ and the continuous dependency of $S^{-1}, \mu$ and $M_{2}$ on $\theta \in \mathcal{S}$. Let $t \in[0, \omega)$. From inequality (A-12) it follows that

$$
\int_{0}^{t}\|z(s)\||\zeta(s)| d s \leq M_{3} \int_{0}^{t}|\zeta(s)|+M_{3} \int_{0}^{t}|\zeta(s)| \int_{0}^{s} e^{-\mu(s-\tau)}|\zeta(\tau)| d \tau d s .
$$

Using the Cauchy-Schwarz inequality, we have that

$$
\int_{0}^{t}|\zeta(s)| \int_{0}^{s} e^{-\mu(s-\tau)}|\zeta(\tau)| d \tau d s \leq\|\zeta(\cdot)\|_{\mathcal{L}^{2}(0, t)} \cdot\left\|\int_{0}^{\bullet} e^{-\mu(\bullet-\tau)}|\zeta(\tau)| d \tau\right\|_{\mathcal{L}^{2}(0, t)} .
$$

An application of the following inequality (Theorem 6.5.54)[V]

$$
\left\|\int_{0}^{\bullet} e^{-\mu(\bullet-\tau)}|\zeta(\tau)| d \tau\right\|_{\mathcal{L}^{2}(0, t)} \leq\left\|e^{-\mu \bullet}\right\|_{\mathcal{L}^{1}(0, t)} \cdot\|\zeta(\cdot)\|_{\mathcal{L}^{2}(0, t)} \leq \mu^{-1}\|\zeta(\cdot)\|_{\mathcal{L}^{2}(0, t)},
$$

together with relations (A-13),(A-8) and (A-14), implies

$$
\begin{aligned}
\int_{0}^{t}\|z(s)\||\zeta(s)| d s & \leq M_{3}\left(1+\mu^{-1}\right) \int_{0}^{t}|\zeta(s)|+\zeta(s)^{2} d s \\
& \leq M_{3}\left(1+\mu^{-1}\right) \int_{0}^{t}\left(1+\eta^{-1}\right)|\zeta(s)||y(s)| d s \\
& \leq M_{4} \hat{\delta}(t),
\end{aligned}
$$

where $M_{4}:=M_{4}\left(x_{0}, \theta\right):=M_{3}\left(1+\mu^{-1}\right)\left(1+\eta^{-1}\right)$. Now, using (A-15), we can calculate a bound on $V(y(t))$ in terms of $\hat{\delta}(t)$ by integrating (A-9) over $[0, t]$

$$
\begin{aligned}
V(y(t)) & \leq V(y(0))+\int_{0}^{t}\left(M_{1}-b_{n-1} \hat{\delta}(s)\right) \dot{\hat{\delta}}(s) d s+\left\|\bar{A}_{2}\right\| M_{4} \hat{\delta}(t) \\
& \leq V_{0}+M_{5} \hat{\delta}(t)-\frac{b_{n-1}}{2} \hat{\delta}(t)^{2}
\end{aligned}
$$


where $V_{0}:=V(y(0))$, and $M_{5}:=M_{5}\left(x_{0}, \theta, d_{\max },\|d(\cdot)\|_{\mathcal{L}^{\infty}}\right):=M_{1}+\left\|\bar{A}_{2}\right\| M_{4}$. The positive definiteness of $V$ implies that

$$
-\frac{b_{n-1}}{2} \hat{\delta}(t)^{2}+M_{5} \hat{\delta}(t)+V_{0} \geq 0
$$

Solving the quadratic inequality $(\mathrm{A}-16)$ for $\hat{\delta}(t)$, we have that

$$
\frac{M_{5}-M_{6}}{b_{n-1}} \leq \hat{\delta}(t) \leq \frac{M_{5}+M_{6}}{b_{n-1}}
$$

where $M_{6}:=M_{6}\left(x_{0}, \theta, d_{\max },\|d(\cdot)\|_{\mathcal{L}^{\infty}}\right):=\sqrt{M_{5}^{2}+2 b_{n-1} V_{0}}>M_{5}$. We discard the nonpositive lower bound of $(\mathrm{A}-17)$ due to the fact that by $(\mathrm{A}-3), \hat{\delta}(0)=0$ and $\hat{\delta}(\cdot)$ is non decreasing. Therefore

$$
0 \leq \hat{\delta}(t) \leq \frac{M_{5}+M_{6}}{b_{n-1}}
$$

The continuous dependency on $x_{0}, \theta, d_{\max }$ of $M_{4}-M_{6}$ is a consequence of the continuous dependency of $M_{3}, \mu$ and the coordinate transformation (6). We now define a continuous function $V^{*}: \mathbb{R}^{n} \times \mathcal{S} \times \mathbb{R}_{\geq 0} \times \mathbb{R}_{>0} \rightarrow \mathbb{R}_{\geq 0}$ by $V^{*}\left(x_{0}, \theta,\|d(\cdot)\|_{\mathcal{L}^{\infty}}, d_{\max }\right)=V_{0}+M_{5}\left(M_{5}+\right.$ $\left.M_{6}\right) / b_{n-1}$. Then

$$
V(y(t)) \leq V^{*}\left(x_{0}, \theta,\|d(\cdot)\|_{\mathcal{L}^{\infty}}, d_{\max }\right) \quad \forall t \in[0, \omega),
$$

The boundedness of $y(\cdot)$ in terms of $V^{*}\left(x_{0}, \theta,\|d(\cdot)\|_{\mathcal{L}^{\infty}}, d_{\max }\right)$ on $[0, \omega)$ follows from (A-4), (A19). Therefore by $(\mathrm{A}-6), \zeta(\cdot)$ is bounded by a continuous function of $V^{*}\left(x_{0}, \theta,\|d(\cdot)\|_{\left.\mathcal{L}^{\infty}, d_{\max }\right)}\right.$ on $[0, \omega)$. Hence by $(\mathrm{A}-12), z(\cdot)$ is bounded by a continuous function of $V^{*}\left(x_{0}, \theta,\|d(\cdot)\|_{\left.\mathcal{L}^{\infty}, d_{\max }\right)}\right.$ on $[0, \omega)$. It follows by $(6)$ that $x(\cdot) \in \mathcal{L}^{\infty}(0, \omega)$ is bounded by a continuous function of $V^{*}\left(x_{0}, \theta,\|d(\cdot)\|_{\mathcal{L}^{\infty}}, d_{\max }\right)$. The continuity of the right hand side of equations (A-1)-(A-3) and the boundedness of the solution $(x(\cdot), \hat{\delta}(\cdot))$ implies that $\omega=\infty$. Finally the boundedness of $u(\cdot)$ as a continuous function of $V^{*}\left(x_{0}, \theta,\|d(\cdot)\|_{\mathcal{L}^{\infty}}, d_{\max }\right)$ follows from (14), thus establishing D1-D2.

Since $y(\cdot)$ is bounded, it follows from the definition of $\hat{\delta}(\cdot)$ that $\dot{\hat{\delta}}(\cdot)$ is uniformly continuous. Furthermore, $\dot{\hat{\delta}}(\cdot) \in \mathcal{L}^{1}$, hence Barbalat's lemma shows $\dot{\hat{\delta}}(t) \rightarrow 0$ as $t \rightarrow \infty$. So $\lim _{t \rightarrow \infty} \inf _{x \in[-\eta, \eta]}|y(t)-x|=0$ hence proving D3, and thus completing the proof.

\section{Appendix B. Proof of Theorem 3.2}

Proof of Theorem 3.2. Since the (time-varying) right hand side of the differential equations (2) and (16) is piecewise locally Lipschitz, a unique piecewise absolutely continuous local solution exists. Let $(x(\cdot), \hat{\delta}(\cdot))$ denote a solution of closed loop $\left(\Sigma\left(x_{0}, \theta, d(\cdot)\right), \Xi_{P}\left(\delta_{\max }\right)\right)$ on a maximum interval of existence $[0, \omega)$ for some $\omega \in[0, \infty)$. Using the transformation defined by (5), we obtain

$$
\begin{aligned}
& \dot{y}(t)=\left(\bar{a}_{1}-b_{n-1} \hat{\delta}(t)\right) y(t)+\bar{A}_{2} z(t)+b_{n-1} d(t), \quad y(0)=C x_{0}, \\
& \dot{z}(t)=\bar{A}_{3} y(t)+\bar{A}_{4} z(t), \quad z(0)=N x_{0}, \\
& \dot{\hat{\delta}}(t)=\left\{\begin{array}{cll}
y(t)^{2} & \hat{\delta}(t)<\delta_{\max } \\
0 & \hat{\delta}(t) \geq \delta_{\max }
\end{array} \quad \hat{\delta}(0)=0\right.
\end{aligned}
$$


By definition of projection modification (16), $\hat{\delta}(t) \leq \delta_{\max }$ for all $t \geq 0$. Let $k_{\theta}^{*}<\delta_{\max }$ be given by equation (10). The monotonicity of $\hat{\delta}(\cdot)$ implies that either $(i) \hat{\delta}(t)<k_{\theta}^{*}$ for all $t \in[0, \omega)$, or $(i i)$ there exists $t^{*} \in[0, \omega)$ such that $\hat{\delta}(t) \geq k_{\theta}^{*}$ for all $t \in\left[t^{*}, \omega\right)$.

(i) If $\hat{\delta}(t)<k_{\theta}^{*}$ for all $t \in[0, \omega)$ then by (16) we have $\dot{\hat{\delta}}(t)=y(t)^{2}$ for all $t \in[0, \omega)$. It follows that

$$
\int_{0}^{\omega} y(s)^{2} d s<k_{\theta}^{*}
$$

and hence $y(\cdot) \in \mathcal{L}^{2}[0, \omega)$. Since $\bar{A}_{4}$ is stable, (B-2) can be considered as an $\mathcal{L}^{2}$ input $y(\cdot)$ to a stable system $\dot{z}(t)=\bar{A}_{4} z(t)+\bar{A}_{3} y(t)$ for all $0 \leq t<\omega$, from which follows that $\dot{z}(\cdot), z(\cdot) \in \mathcal{L}^{2}[0, \omega)$. An explicit bound on $\|z\|_{\mathcal{L}^{2}[0, \omega)}$ can be obtained as follows. Note that since $y(\cdot) \in \mathcal{L}^{2}[0, \omega)$ and $\bar{A}_{4}$ is exponentially stable, by Lemma A-1, there exists $M_{0}, \nu \geq 0$ such that for all $t \in[0, \omega)$

$$
M_{0} e^{-\nu t} \geq\left\|e^{\bar{A}_{4} t}\right\| \quad M_{0}:=M_{0}(\theta)=\sqrt{\bar{\lambda}(R) / \underline{\lambda}(R)}, \quad \nu:=\nu(\theta)=1 / \bar{\lambda}(R),
$$

where $R$ is defined in (8). Therefore ${ }^{2}$

$$
\begin{aligned}
\|z\|_{\mathcal{L}^{2}[0, \omega)} & \leq\left\|e^{\bar{A}_{4} \bullet} z_{0}\right\|_{\mathcal{L}^{2}[0, \omega)}+\left\|\left(s I-\bar{A}_{4}\right)^{-1} \bar{A}_{3}\right\|_{H_{\infty}}\|y\|_{\mathcal{L}^{2}[0, \omega)} \\
& \leq \frac{M_{0}}{\nu}\left\|z_{0}\right\|+\left\|\left(s I-\bar{A}_{4}\right)^{-1} \bar{A}_{3}\right\|_{H_{\infty}} \sqrt{k_{\theta}^{*}}
\end{aligned}
$$

A bound on $z(\cdot)$ is also required to show the boundedness of $x(\cdot)$. Applying (B-4) to the solution of (A-2), we have that for all $t \in[0, \omega)$,

$$
\begin{aligned}
\|z(t)\| & \leq \frac{M_{0}}{\nu}\left\|z_{0}\right\|+M_{0} \int_{0}^{t} e^{-\nu(t-s)}\left\|\bar{A}_{3}\right\||y(s)| d s \\
& \leq M_{1}\left(1+\int_{0}^{t} e^{-\nu(t-s)}|y(s)| d s\right),
\end{aligned}
$$

where $M_{1}:=M_{1}\left(\left\|x_{0}\right\|, \theta\right):=M_{0}\left(\frac{1}{\nu}\left\|z_{0}\right\|+\left\|\bar{A}_{3}\right\|\right)$. Since $y(\cdot) \in \mathcal{L}^{2}[0, \omega)$, and $\|y\|_{\mathcal{L}^{2}[0, \omega)} \leq$ $k_{\theta}^{*}$, it follows that $z(\cdot)$ is bounded as a continuous function of $\left\|x_{0}\right\|$ and $\theta$. It remains to show $y(\cdot) \in \mathcal{L}^{\infty}[0, \omega)$, and to construct an explicit uniform bound for $y(\cdot)$ which is continuously dependent on $x_{0}, \theta,\|d\|, \delta_{\max }$. To this end, consider (A-1) and define:

$$
V_{1}(t):=\frac{1}{2} y(t)^{2}, \quad \forall t \in[0, \omega) .
$$

The time derivative of (B-7) along the solution of (B-1)- (B-3) is bounded by:

$$
\begin{aligned}
\dot{V}_{1}(t) & =\left(\bar{a}_{1}-b_{n-1} \hat{\delta}(t)\right) y(t)^{2}+\bar{A}_{2} z(t) y(t)+b_{n-1} d(t) y(t) \\
& =-\frac{1}{2} y(t)^{2}+\left(\bar{a}_{1}+1\right) y(t)^{2}-b_{n-1} \hat{\delta}(t) y(t)^{2}+\bar{A}_{2} z(t) y(t)-\frac{1}{2} y(t)^{2}+b_{n-1} d(t) y(t) \\
& \leq-V_{1}(t)+\left(\bar{a}_{1}+1+\left\|\bar{A}_{2}\right\|^{2}\right) y(t)^{2}+|z(t)|^{2}+\frac{b_{n-1}^{2}}{2}\|d(\cdot)\|_{\mathcal{L}^{\infty}}^{2}, \quad \forall t \in[0, \omega) \quad(\mathrm{B}-8)
\end{aligned}
$$

\footnotetext{
${ }^{2}$ Recall that $\|G(\cdot)\|_{H^{\infty}}=\operatorname{ess} \sup _{\omega \in \mathbb{R}}|G(j w)|$.
} 
where (B-8) follows from two applications of Young's inequality and by observing that $b_{n-1} \hat{\delta}(t)>0$ for all $t \in[0, \omega)$. Hence for all $t \in[0, \omega)$,

$$
\begin{aligned}
\frac{1}{2} y(t)^{2} & \leq V_{1}(t) \\
& \leq e^{-t} V_{1}(0)+\int_{0}^{t} e^{-(t-s)}\left(\left(\bar{a}_{1}+1+\left\|\bar{A}_{2}\right\|^{2}\right) y(s)^{2}+|z(s)|^{2}+\frac{b_{n-1}^{2}}{2}\|d(\cdot)\|_{\mathcal{L}^{\infty}}^{2}\right) \mathrm{ds} \\
& \leq \frac{y(0)^{2}}{2}+\left(\bar{a}_{1}+1+\left\|\bar{A}_{2}\right\|^{2}\right)\|y(\cdot)\|_{\mathcal{L}^{2}[0, \omega)}^{2}+\|z(\cdot)\|_{\mathcal{L}^{2}[0, \omega)}^{2}+\frac{b_{n-1}^{2}}{2}\|d(\cdot)\|_{\mathcal{L}^{\infty} \cdot(\mathrm{B}-9)}^{2}
\end{aligned}
$$

Since $\|y(\cdot)\|_{\mathcal{L}^{2}[0, \omega)}^{2}<k_{\theta}^{*}$ which is continuously dependent on $\theta$, and we have shown that $\|z(\cdot)\|_{\mathcal{L}^{2}[0, \omega)}^{2}$ is bounded as a continuous function of $\left|x_{0}\right|$ and $\theta$, it follows that $y(\cdot)$ is bounded on $[0, \omega)$ in terms of a continuous function of $x_{0}, \theta,\|d\|$ and $\delta_{\max }$. It follows by (B-6) and (6) that $x(\cdot)$ is bounded on $[0, \omega)$ as a continuous function of $x_{0}, \theta,\|d\|, \delta_{\max }$.

(ii) Suppose there exists $t^{*} \in[0, \omega)$ such that $\hat{\delta}(t) \geq k_{\theta}^{*}$ for all $t \in\left[t^{*}, \omega\right)$. Define the Lyapunov function

$$
V_{2}(t)=\bar{x}(t)^{T} P \bar{x}(t), \quad \forall t \in[0, \omega)
$$

where $\bar{x}(t), P$ are defined by (6) and (9) respectively.

Then all $t \in[0, \omega)$, the time derivative of $V_{2}(t)$ w.r.t. (B-1)- (B-3) is given by:

$$
\begin{aligned}
\dot{V}_{2}(t) & =\bar{x}(t)^{T} P \dot{\bar{x}}(t)+\dot{\bar{x}}(t)^{T} P \bar{x}(t), \\
& =\bar{x}(t)^{T} P(\bar{D}(\hat{\delta}(t)) \bar{x}(t)+\bar{B} d(t))+(\bar{D}(\hat{\delta}(t)) \bar{x}+\bar{B} d(t))^{T} P \bar{x}(t), \\
& =\bar{x}(t)^{T}\left(P \bar{D}(\hat{\delta}(t))+\bar{D}^{T}(\hat{\delta}(t)) P\right) \bar{x}(t)+2 \bar{x}(t)^{T} P \bar{B} d(t),
\end{aligned}
$$

where $S$ is given by $(5)$ and $\bar{B}:=S^{-1} B, \bar{D}(\bullet):=S^{-1}(A-\bullet B C) S$. Let

$$
\mu(\theta,\|d\|):=\frac{2 \sqrt{\underline{\lambda}(P)}}{\underline{\lambda}(Q)}|P \bar{B}|\|d(\cdot)\|_{\mathcal{L}^{\infty}},
$$

where $Q$ is defined in (9). Then $\mu$ is continuously dependent on $\theta \in \mathcal{S}$ by the continuity of the transformation (6). By (9), we observe that

$$
\begin{aligned}
\dot{V}_{2}(t) & \leq-\bar{x}(t)^{T} Q \bar{x}(t)+2 \bar{x}(t)^{T} P \bar{B} d(t), \\
& \leq-\left(\underline{\lambda}(Q)-\frac{2 \sqrt{\underline{\lambda}(P)}}{\sqrt{V_{2}(t)}}|P \bar{B}|\|d(\cdot)\|_{\mathcal{L}^{\infty}}\right)\|\bar{x}(t)\|^{2}, \\
& \leq-\underline{\lambda}(Q)\left(1-\frac{\mu(\theta,\|d\|)}{\sqrt{V_{2}(t)}}\right)\|\bar{x}(t)\|^{2}, \quad \forall t \in\left[t^{*}, \omega\right) .
\end{aligned}
$$

Inequality (B-14) implies that $\dot{V}_{2}(t)<0$ for all $V_{2}(t)>\mu(\theta,\|d\|)^{2}$, i.e.

$$
V(t) \leq V^{\prime}\left(\bar{x}^{*}, \theta,\|d\|\right):=\max \left\{V_{2}\left(\bar{x}^{*}\right), \mu(\theta,\|d\|)^{2}\right\} \quad \forall t \in\left[t^{*}, \omega\right),
$$

where $\bar{x}^{*}:=\bar{x}\left(t^{*}\right)$. Therefore, by $(\mathrm{B}-10), \bar{x}(\cdot)$ is bounded on $\left[t^{*}, \omega\right)$ in terms of $V^{\prime}\left(\bar{x}^{*}, \theta,\|d\|\right)$. A bound on $\bar{x}(\cdot)$ on $\left[0, t^{*}\right)$ and a bound on the end point $\bar{x}\left(t^{*}\right)$ as a continuous function of $x_{0}, \theta,\|d\|, \delta_{\max }$ follows directly by the argument of part $(i)$ where $\hat{\delta}(t)<k_{\theta}^{*}<\delta_{\max }$ for all $t \in\left[0, t^{*}\right)$. Hence by the continuity of the transformation $(6), x(\cdot)$ is bounded on $[0, \omega)$ as a continuous function of $x_{0}, \theta,\|d\|, \delta_{\max }$. 
The boundedness of $x(\cdot)$ over $[0, \omega)$ implies that $x(\cdot)$ cannot have a finite escape time. By definition $(16), \hat{\delta}(\cdot)$ is also known never to leave the set $\left[0, \delta_{\max }\right]$. Hence $\omega=\infty$ i.e. the solution $(x(\cdot), \hat{\delta}(\cdot))$ exists for all $t \in[0, \infty)$. Furthermore, the solution is bounded as a continuous function of $x_{0}, \theta,\|d\|, \delta_{\max }$. Finally, the boundedness of $u(\cdot)$ as a continuous function of $x_{0}, \theta,\|d\|, \delta_{\max }$ follows by (16).

\section{References}

[E] B. Egardt. Stability of adaptive controllers. Lecture notes in Control and Information Sciences, Springer-Verlag, New York, 1979.

[F] M. French. An analytical comparison between the nonsingular quadratic performance of robust and adaptive backstepping designs. IEEE Trans. on Automatic Control, 47(4):670-675, 2002.

[GI] P. Georgieva and A. Ilchmann. Adaptive $\lambda$-tracking control of activated sludge processes. International Journal of Control, 74:1247-1259, 2001.

[I1] A. Ilchmann. Non-Identifier-Based Adaptive Control of Dynamical systems: A Survey. IMA Journal of Mathematical Control and Information,8:321-366, 1991.

[I2] A. Ilchmann. Non-Identifier-Based High-Gain Adaptive Control. Lecture notes in Control and Information Sciences, Springer-Verlag, London, 1993.

[IR] A. Ilchmann and E. P. Ryan. Universal $\lambda$-tracking for nonlinearly perturbed systems in the presence of noise. Automatica, 30:337-346, 1994.

[IT] A. Ilchmann and S. Townley. Adaptive sampling control of high-gain stabilizable systems. IEEE Trans. on Automatic Control, 44(10):1961-1966, 1999.

[KKK] M. Kristic, I. Kanellakopoulos, and P. Kokotovic. Nonlinear and Adaptive Control Design. John Wiley \& Sons, New York, 1995.

$[\mathrm{KN}] \quad$ G. Kreisselmeier and K. S. Narendra. Stable model reference adaptive control in the presence of bounded disturbances. IEEE Trans. on Automatic Control, 27(6):1169-1175, 1982.

[LK] K. W. Lee and H. K. Khalil. Adaptive output feedback control of robot manipulators using high-gain observer. Int. Journal of Control, 67(6):869-886, 1997.

[LT] H. Logemann and S. Townley. Adaptive stabilization without identification for distributed parameter systems: An overview. IMA Journal Mathematical Control and Information, 20:175-206, 1997.

[M] B. Mårtensson. The order of any stabilizing regulator is sufficient a priori information for adaptive stabilization. Systems and Control Letters, 6:87-91, 1985.

[NA] K. S. Narendra and A. M. Annaswamy. Stable Adaptive Systems. Prentice-Hall, 1989 .

[PN] B. B. Peterson and K. S. Narendra. Bounded error adaptive control. IEEE Trans. on Automatic Control, 27(6):1161-1168, 1982. 
[R] E. P. Ryan. A universal adaptive stabilizer for class of nonlinear systems. Systems and Control Letters, 16:209-218, 1991.

[S] A. Sanei. Towards a Performance Theory of Robust Adaptive Control. PhD thesis, Department of Electronics and Computer Science, University of Southampton, U.K., 2002.

[SF] A. Sanei and M. French. Towards a performance theory of robust adaptive control. International Journal of Adaptive Control and Signal Processing, 18:403-421, 2004.

[T] S. Townley. Topological aspects of universal adaptive stabilization. SIAM Journal Control and Optimization, 34:1044-1070, 1996.

[V] M. Vidyasagar. Nonlinear Systems Analysis. Prentice-Hall, 1978.

[W] A. J. Weir. Lebesgue Integration and Measure. Cambridge University Press, 1991.

[WB] J. C. Willems and C. I. Byrnes. Global adaptive stabilization in the absence of information on the sign of the high-frequency gain. In Lecture Notes in control and information science, Springer-Verlag, Berlin, 62:49-57, 1984.

[XF] C. Xie and M. French. A performance comparison between two design techniques for non-linear output feedback control. International Journal of Control, 77:264$276,2004$. 